\title{
DYNAMICAL MODELING AND COUPLING CHARACTERISTIC ANALYSIS OF THE MULTISTAGE GEARS SYSTEM IN A CONTINUOUS MINER
}

\author{
WENJIA LU \\ School of Mechanical Engineering and Automation, Northeastern University, Shenyang, China \\ YIMIN ZHANG \\ College of Mechanical Automotive Engineering, Zhaoqing University, Zhaoqing, China, and \\ Equipment Reliability Institute, Shenyang University of Chemical Technology, Shenyang, China \\ e-mail:zhangyimin@syuct.edu.cn \\ Hongchuan Cheng, Zhou Yang \\ School of Mechanical Engineering and Automation, Northeastern University, Shenyang, China
}

\begin{abstract}
The lumped parameter method is adopted to establish a dynamic model of two stage planets and multistage fixed-shaft gears in which the effect of coupling shaft section radius on the system in a continuous miner is considered. The acceleration test of the system proves the accuracy of the proposed model. With the increase of cross section size of the coupled shaft, the influence of the first stage meshing frequency on the second stage planetary system is enhanced. The acceleration decreases gradually with the increase of the size of coupled carrier. It is shown that the modeling by considering the coupled size contributes to reduction of the vibration level.
\end{abstract}

Keywords: planets, multistage gear, continuous miner, acceleration

\section{Nomenclature}

The subscripts $c, r, s, p$ represent planet carrier, ring gear, sun gear and planet gear, respectively. $i=1,2$ denotes the number of stages of the planetary gears subsystem, $n=1,2,3,4$ is the index of planet gear.

$g_{m}(m=1,2, \ldots, 8)$ - gear in multistage fixed-shaft subsystem,

$\delta_{\text {spni }}, \delta_{\text {rpni }}$ - relative displacement between sun (ring) and $n$-th planet along mesh line,

$\delta_{p n \zeta i}, \delta_{p n \eta i}$ - relative displacement between carrier and $n$-th planet in direction of $\zeta$ and $\eta$,

$\delta_{j k}, \psi_{j k}, k_{j k}, c_{j k}$ - relative displacement of gear pairs along mesh line, angle between $y$-axis and mesh line, mesh stiffness and damping for gear $j$ and gear $k$ in fixed-shaft gears subsystem, respectively, where $(j, k)=(1,2),(2,3),(4,5),(5,6),(6,7),(7,8)$,

$m_{j}, I_{j}, r_{j}$ - mass, moment of inertia and base radius of gear $j(j=1,2, \ldots, 8)$, respectively,

$\psi_{n i}-n$-th planet position angle,

$\alpha_{s i}, \alpha_{r i}$ - pressure angles of sun and ring gear,

$\delta_{\text {ex12 }}, \delta_{\text {ey12 }}, \delta_{\text {eu12 }}$ - relative displacement of the coupling element between two stage planets in corresponding directions,

$\delta_{m n x}, \delta_{m n y}, \delta_{m n u}$ - relative displacement of coupling element between first planets stage and low speed spur gears stage in corresponding directions,

$\delta_{34 x}, \delta_{34 y}, \delta_{34 u}$ - relative displacement of coupling element between low speed spur gears stage and high speed spur gears stage in corresponding directions, 
$r_{e}, r_{m n}, r_{34}$ - connecting shafts radius of two-stage planets, first planets stage and low speed spur gears stage, low speed spur gears stage and high speed spur gears stage, respectively, $m_{s i}, m_{r i}, m_{c i}, m_{p n i}$ - mass of sun, ring, carrier and planet, respectively,

$I_{s i}, I_{r i}, I_{c i}, I_{p n i}$ - moment of inertia of sun, ring, carrier and planet, respectively,

$r_{s i}, r_{r i}, r_{c i}, r_{p n i}$ - base radius of sun, ring, carrier and planet, respectively,

$k_{\text {spni }}, k_{\text {rpni }}, c_{\text {spni }}, c_{r p n i}$ - meshing stiffness and damping in planetary system,

$k_{p n \zeta i}, k_{p n \eta i}, c_{p n \zeta i}, c_{p n \eta i}$ - support stiffness and damping of planet gears in $\zeta$ and $\eta$ directions, respectively,

$k_{s x i}, k_{s y i}, c_{s x i}, c_{s y i}$ - support stiffness and damping of sun gear in $x$ and $y$ directions, respectively,

$k_{r x i}, k_{r y i}, c_{r x i}, c_{r y i}$ - support stiffness and damping of ring gear in $x$ and $y$ directions, respectively,

$k_{c x i}, k_{c y i}, c_{c x i}, c_{c y i}$ - support stiffness and damping of planet carrier in $x$ and $y$ directions, respectively,

$k_{\text {sui }}, k_{\text {rui }}, k_{\text {cui }}, c_{\text {sui }}, c_{\text {rui }}, c_{c u i}$ - torsional stiffness and damping in planetary system,

$\mathbf{M}, \mathbf{C}, \mathbf{K}_{b}, \mathbf{K}_{m}(t)$ - mass matrix, damping matrix and support stiffness matrix and mesh stiffness matrix in coupling system, respectively,

$\mathbf{q}, \mathbf{F}(t)$ - displacement vector and load vector, respectively,

$\mathbf{M}_{p 1}, \mathbf{M}_{p 2}, \mathbf{M}_{l}, \mathbf{M}_{h}$ - mass matrices of first planet stage, second planet stage, low speed spur gears stage and high speed spur gears stage, respectively,

$\mathbf{K}_{b p i}, \mathbf{K}_{b l}, \mathbf{K}_{b h}$ - support stiffness matrices of planet stages, low speed spur gears stage and high speed spur gears stage, respectively,

$\mathbf{K}_{m p i}(t), \mathbf{K}_{m l}(t), \mathbf{K}_{m h}(t)$ - time-varying meshing stiffness matrices for planet stages, low-speed and high-speed spur gears stage, respectively,

$\mathbf{K}_{p 1 p 2}, \mathbf{K}_{p 1 l}, \mathbf{K}_{l h}$ - coupling stiffness matrices of two planetary stages, first-stage planetary and low-speed spur gears stage, low-speed spur gears stage and high-speed spur gears stage, respectively.

\section{Introduction}

The multistage gears system studied in this paper is formed by coupling of a two stages planetary gears subsystem and a multistage fixed-shaft spur gears subsystem. As for research of the planetary gears system, Lin and Parker (1999) and Eritenel and Parker (2009) proposed a dynamic analysis model of the planetary system using a lumped parameter method, and analyzed the frequency property and vibration characteristics in the planetary system, which laid a foundation for the future research of planetary gears. Karray et al. (2013), Hammami et al. (2015a,b, 2016) developed a test device suitable for dynamic behavior research, and carried out theoretical modeling and model verification by a back-to-back planetary gears system. The effects of load and speed on the dynamic behavior of the planetary gears were studied respectively. Due to the change of mesh stiffness in the planetary gears set under different load conditions, the internal excitation is changed in the system and the influence of variable load on the planetary system is analyzed. At the same time, the dynamic behavior of the system is studied in the process of speed change at the moment of starting and stopping. Liu et al. (2016), Bai et al. (2018) and Yi et al. (2018) studied the interaction mechanism between the planetary gear transmission system and motor, established the electromechanical dynamics model of the multistage gear transmission system considering the electromagnetic induction characteristics of the motor, analyzed and pointed out that the electromechanical coupling effect would aggravate the vibration level of the system, and proposed the speed regulation and monitoring methods to improve the electromechanical performance of the system. The coupled modeling approach of 
multistage planetary gears system was proposed by Wei et al. (2017) and Zhang et al. (2017). The fully coupled dynamic modeling method combines the lumped parameter method with the finite element method, which improves the modeling speed and accuracy. The analytical model of the planetary gears system including wedge and bearing clearance was established by Guo and Parker $(2010,2012)$. The relationship between the bearing force and tooth force of planet gears was studied by analyzing the physical force and displacement acting on the whole planetary gears. The prediction method of wedge was developed and validated based on geometric interaction. Liu et al. $(2014,2017)$ proposed a analytical approach for dynamic behavior of the planetary system in a variable speed process and high speed process with the centrifugal force taken into account. The influence of tooth profile error excitation on vibration and the dynamic meshing force was studied before and after gear separation, and corresponding suggestions were put forward in terms of restraining system vibration and reducing the dynamic meshing force. In the research of a fixed-shaft gear system, Kubur et al. (2004) developed an analytical model of a multistage gear transmission system by shaft finite elements. The model also considers the influence of bearing stiffness. The vibration modes of the gear set were predicted by eigenvalue solution and a modal summation technique. Chowdhury anf Yedavalli (2017, 2018) studied the dynamic behavior of a fixed-shaft gear system mounted on rigid bearings at low and high speeds, analyzed the sensitivity of natural frequencies to different gear parameters, obtaining the response of the meshing process caused by a static transmission error. Ma et al. $(2012,2013)$ developed a general gear meshing dynamics model with full degree of freedom, and analyzed the effect of gear transmission error, unbalanced rotor mass, geometric eccentricity and their coupling on the dynamic behavior of the system.

\section{Dynamics modeling of a multistage gear system}

The multistage gear system is composed of a multistage fixed shaft gear subsystem and a two-stage planetary gear subsystem. Figure 1 is a three-dimensional model of the coupled gear system in the cutting section of a continuous miner. Figure 2 shows the kinematic scheme of the coupled gear system.

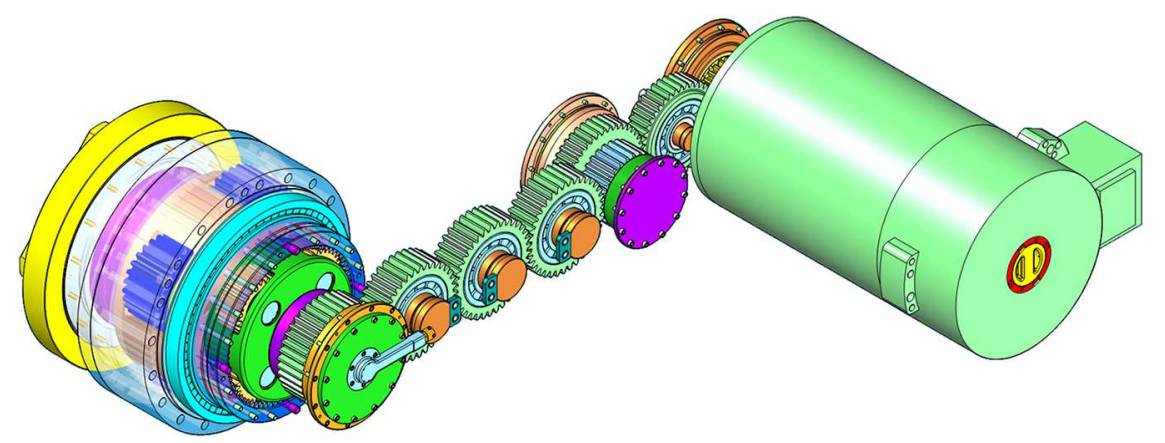

Fig. 1. The multistage gear system in the cutting section of a continuous miner

Figure 3 is the translation-torsion coupling dynamic model of a multistage gear transmission system. The system model is divided into a high speed spur gears stage, low speed spur gears stage, first planets stage and the second planets stage. The spring damper system simulates the supporting effect of the gear system in the $x$ and $y$ directions. The meshing action between gear teeth is simulated by the spring and damper system in the direction of the meshing line. Different subsystems are connected by the elastic shaft. The influence of coupling components radius on the torsional moment is fully considered in the coupling system. Figure 4 shows the position relationship of planetary gears in the two-stage planetary subsystem. 


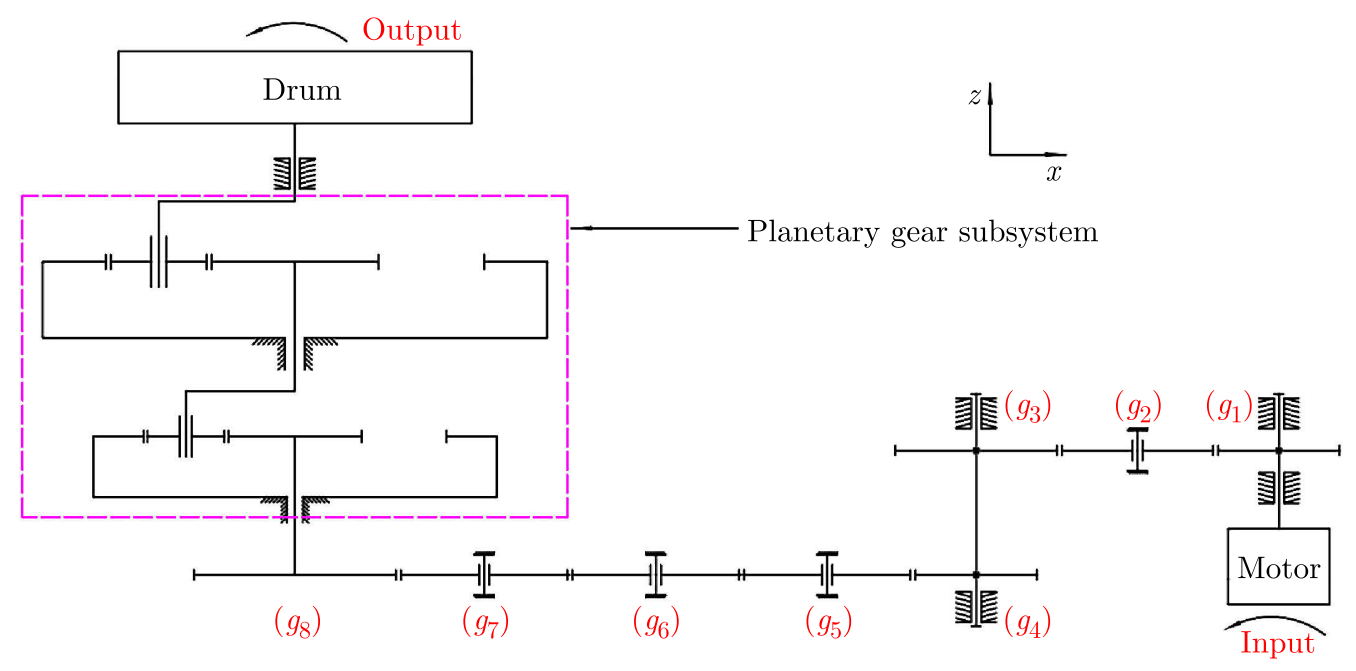

Fig. 2. Kinematic scheme of the coupled gear system

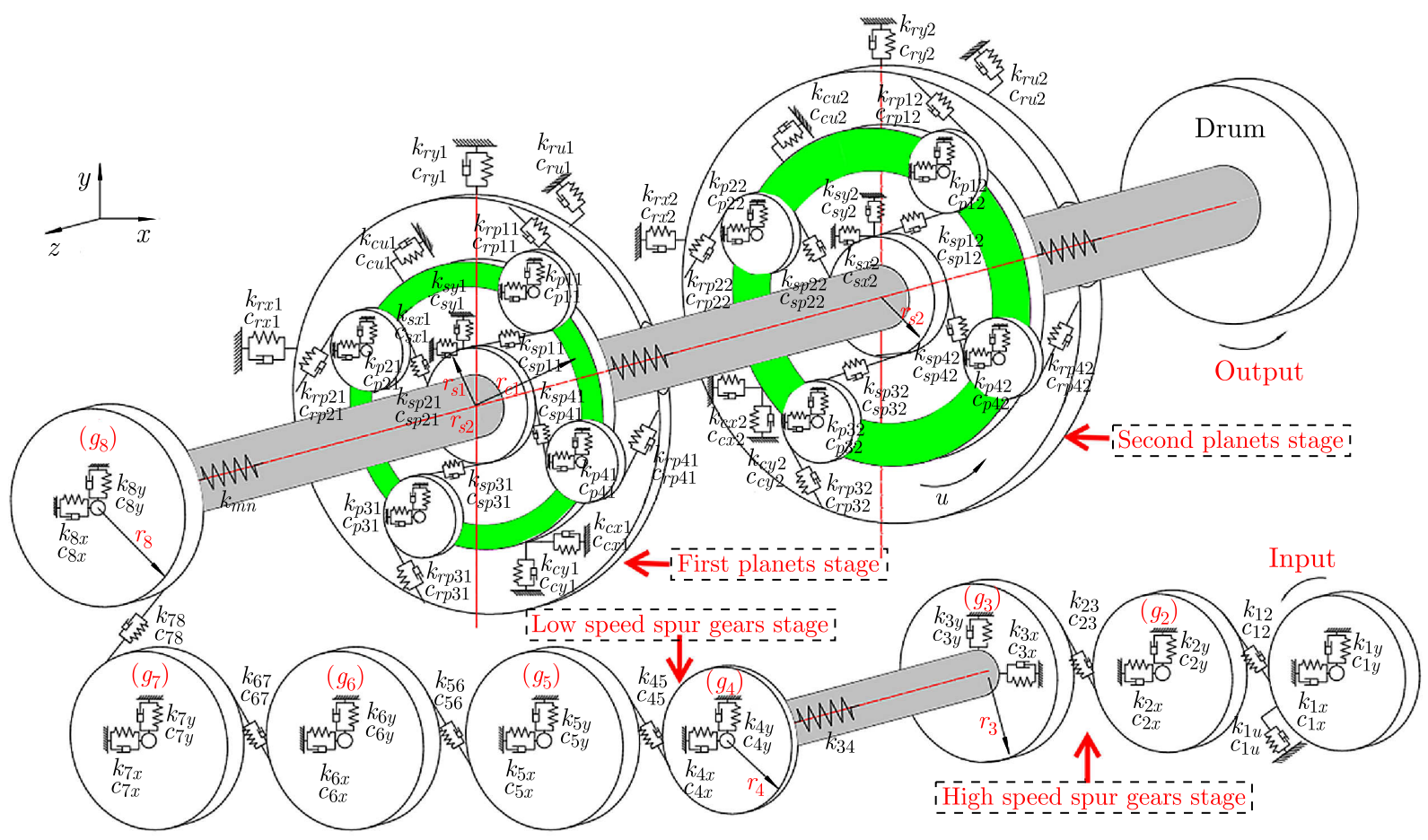

Fig. 3. The coupling dynamic model of the multistage gear transmission system

The relative displacements of the system associated components are as follows $(i=1,2)$

$$
\begin{aligned}
& \delta_{s p n i}=-x_{s i} \sin \psi_{s n i}+y_{s i} \cos \psi_{s n i}-\eta_{p n i} \cos \alpha_{s i}-\zeta_{p n i} \sin \alpha_{s i}+u_{s i}+u_{p n i} \\
& \delta_{r p n i}=-x_{r i} \sin \psi_{r n i}+y_{r i} \cos \psi_{r n i}+\eta_{p n i} \cos \alpha_{r i}-\zeta_{p n i} \sin \alpha_{r i}+u_{r i}-u_{p n i} \\
& \delta_{p n \zeta i}=x_{c i} \cos \psi_{n i}+y_{c i} \sin \psi_{n i}-\zeta_{p n i} \\
& \delta_{p n \eta i}=-x_{c i} \sin \psi_{n i}+y_{c i} \cos \psi_{n i}+u_{c i}-\eta_{p n i} \\
& \delta_{j k}=\left(-x_{j}+x_{k}\right) \sin \psi_{j k}+\left(y_{j}-y_{k}\right) \cos \psi_{j k}+u_{j}+u_{k}
\end{aligned}
$$




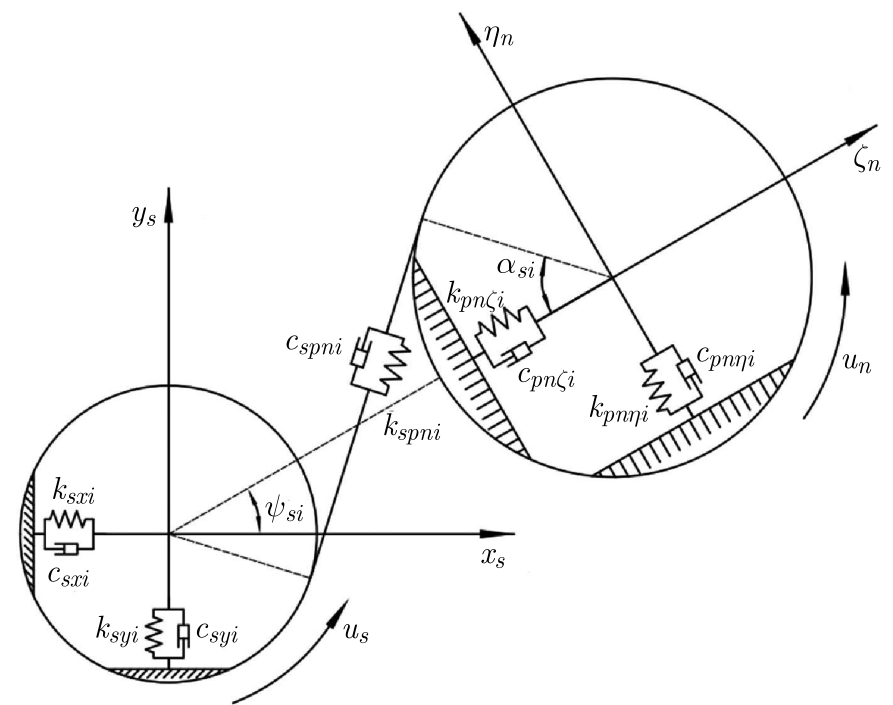

Fig. 4. Position relationship of planetary gears in the two-stage planetary subsystem

The system is coupled by connecting shafts. Considering the influence of coupling shaft radius, the relative displacements of the connecting parts between the stages are as follows

$$
\begin{aligned}
\delta_{e x 12}=x_{c 1}-x_{s 2} & \delta_{e y 12}=y_{c 1}-y_{s 2} & \delta_{e u 12}=u_{c 1} \frac{r_{e}}{r_{c 1}}-u_{s 2} \frac{r_{e}}{r_{s 2}} \\
\delta_{m n x}=x_{s 1}-x_{s} & \delta_{m n y}=y_{s 1}-y_{s} & \delta_{m n u}=u_{s 1} \frac{r_{m n}}{r_{s 1}}-u_{s} \frac{r_{m n}}{r_{s}} \\
\delta_{34 x}=x_{3}-x_{4} & \delta_{34 y}=y_{3}-y_{4} & \delta_{34 u}=u_{3} \frac{r_{34}}{r_{3}}-u_{4} \frac{r_{34}}{r_{4}}
\end{aligned}
$$

Referring to the dynamic modeling method of a single-stage planetary system by Lin and Parker (1999), considering the stiffness of coupling shaft in the $x, y$ and $u$ directions, a two-stage planetary system equation of dynamics are established.

The differential equations of motion of the ring gear are $(i=1,2)$

$$
\begin{aligned}
& m_{r i} \ddot{x}_{r i}-\sum_{n=1}^{4} k_{r p n i} \delta_{r p n i} \sin \psi_{r n i}-\sum_{n=1}^{4} c_{r p n i} \dot{\delta}_{r p n i} \sin \psi_{r n i}+k_{r x i} x_{r i}+c_{r x i} \dot{x}_{r i}=0 \\
& m_{r i} \ddot{y}_{r i}+\sum_{n=1}^{4} k_{r p n i} \delta_{r p n i} \cos \psi_{r n i}+\sum_{n=1}^{4} c_{r p n i} \dot{\delta}_{r p n i} \cos \psi_{r n i}+k_{r y i} y_{r i}+c_{r y i} \dot{y}_{r i}=0 \\
& \frac{I_{r i}}{r_{r i}^{2}} \ddot{u}_{r i}-\sum_{n=1}^{4} k_{r p n i} \delta_{r p n i}-\sum_{n=1}^{4} c_{r p n i} \dot{\delta}_{r p n i}+k_{r u i} u_{r i}+c_{r u i} \dot{u}_{r i}=0
\end{aligned}
$$

The differential equations of motion for the planet gear are $(i=1,2)$

$$
\begin{aligned}
& m_{p n i} \ddot{\zeta}_{p n i}-k_{s p n i} \delta_{s p n i} \sin \alpha_{s i}-c_{s p n i} \dot{\delta}_{s p n i} \sin \alpha_{s i}-k_{r p n i} \delta_{r p n i} \sin \alpha_{r i} \\
& -c_{r p n i} \dot{\delta}_{r p n i} \sin \alpha_{r i}-k_{p n \zeta i} \delta_{p n \zeta i}-c_{p n \zeta i} \dot{\delta}_{p n \zeta i}=0 \\
& m_{p n i} \ddot{\eta}_{p n i}-k_{s p n i} \delta_{s p n i} \cos \alpha_{s i}-c_{s p n i} \dot{\delta}_{s p n i} \cos \alpha_{s i}-k_{r p n i} \delta_{r p n i} \cos \alpha_{r i} \\
& -c_{r p n i} \dot{\delta}_{r p n i} \cos \alpha_{r i}-k_{p n \eta i} \delta_{p n \eta i}-c_{p n \eta i} \dot{\delta}_{p n \eta i}=0 \\
& \frac{I_{p n i}}{r_{p n i}^{2}} \ddot{u}_{p n i}+k_{s p n i} \delta_{s p n i}+c_{s p n i} \dot{\delta}_{s p n i}-k_{r p n i} \delta_{r p n i}-c_{r p n i} \dot{\delta}_{r p n i}=0
\end{aligned}
$$


The differential equations of motion for the second-stage planet carrier are

$$
\begin{aligned}
& m_{c 2} \ddot{x}_{c 2}+\sum_{n=1}^{4} k_{p n \zeta 2}\left(\delta_{p n \zeta 2} \cos \psi_{n 2}-\delta_{p n \eta 2} \sin \psi_{n 2}\right) \\
& \quad+\sum_{n=1}^{4} c_{p n \zeta 2}\left(\dot{\delta}_{p n \zeta 2} \cos \psi_{n 2}-\dot{\delta}_{p n \eta 2} \sin \psi_{n 2}\right)+k_{c x 2} x_{c 2}+c_{c x 2} \dot{x}_{c 2}=0 \\
& m_{c 2} \ddot{y}_{c 2}+\sum_{n=1}^{4} k_{p n \eta 2}\left(\delta_{p n \zeta 2} \sin \psi_{n 2}+\delta_{p n \eta 2} \cos \psi_{n 2}\right) \\
& \quad+\sum_{n=1}^{4} c_{p n \eta 2}\left(\dot{\delta}_{p n \zeta 2} \sin \psi_{n 2}+\dot{\delta}_{p n \eta 2} \cos \psi_{n 2}\right)+k_{c y 2} y_{c 2}+c_{c y 2} \dot{y}_{c 2}=0 \\
& \frac{I_{c 2}}{r_{c 2}^{2}} \ddot{u}_{c 2}+\sum_{n=1}^{4} k_{p n \eta 2} \delta_{p n \eta 2}+\sum_{n=1}^{4} c_{p n \eta 2} \dot{\delta}_{p n \eta 2}+k_{c u 2} u_{c 2}+c_{c u 2} \dot{u}_{c 2}=-\frac{T_{c 2}}{r_{c 2}}
\end{aligned}
$$

The dynamic differential equations for the second-stage sun gear are

$$
\begin{aligned}
& m_{s 2} \ddot{x}_{s 2}-\sum_{n=1}^{4} k_{s p n 2} \delta_{s p n 2} \sin \psi_{s n 2}-\sum_{n=1}^{4} c_{s p n 2} \dot{\delta}_{s p n 2} \sin \psi_{s n 2} \\
& \quad+k_{s x 2} x_{s 2}+c_{s x 2} \dot{x}_{s 2}+k_{e x 12} \delta_{e x 12}+c_{e x 12} \dot{\delta}_{e x 12}=0 \\
& m_{s 2} \ddot{y}_{s 2}+\sum_{n=1}^{4} k_{s p n 2} \delta_{s p n 2} \cos \psi_{s n 2}+\sum_{n=1}^{4} c_{s p n 2} \dot{\delta}_{s p n 2} \cos \psi_{s n 2} \\
& \quad+k_{s y 2} y_{s 2}+c_{s y 2} \dot{y}_{s 2}+k_{e y 12} \delta_{e y 12}+c_{e y 12} \dot{\delta}_{e y 12}=0 \\
& \frac{I_{s 2}}{r_{s 2}^{2}} \ddot{u}_{s 2}+\sum_{n=1}^{4} k_{s p n 2} \delta_{s p n 2}+\sum_{n=1}^{4} c_{s p n 2} \dot{\delta}_{s p n 2}+k_{s u 2} u_{s 2}+c_{s u 2} \dot{u}_{s 2} \\
& \quad+k_{e u 12} \delta_{e u 12}+c_{e u 12} \dot{\delta}_{e u 12}=0
\end{aligned}
$$

The dynamic differential equations for the first-stage planet carrier are

$$
\begin{aligned}
& m_{c 1} \ddot{x}_{c 1}+\sum_{n=1}^{4} k_{p n \zeta 1}\left(\delta_{p n \zeta 1} \cos \psi_{n 1}-\delta_{p n \eta 1} \sin \psi_{n 1}\right) \\
& \quad+\sum_{n=1}^{4} c_{p n \zeta 1}\left(\dot{\delta}_{p n \zeta 1} \cos \psi_{n 1}-\dot{\delta}_{p n \eta 1} \sin \psi_{n 1}\right) \\
& \quad+k_{c x 1} x_{c 1}+c_{c x 1} \dot{x}_{c 1}-k_{e x 12} \delta_{e x 12}-c_{e x 12} \dot{\delta}_{e x 12}=0 \\
& m_{c 1} \ddot{y}_{c 1}+\sum_{n=1}^{4} k_{p n \eta 1}\left(\delta_{p n \zeta 1} \sin \psi_{n 1}+\delta_{p n \eta 1} \cos \psi_{n 1}\right) \\
& \quad+\sum_{n=1}^{4} c_{p n \eta 1}\left(\dot{\delta}_{p n \zeta 1} \sin \psi_{n 1}+\dot{\delta}_{p n \eta 1} \cos \psi_{n 1}\right) \\
& \quad+k_{c y 1} y_{c 1}+c_{c y 1} \dot{y}_{c 1}-k_{e y 12} \delta_{e y 12}-c_{e y 12} \dot{\delta}_{e y 12}=0 \\
& \frac{I_{c 1}}{r_{c 1}^{2}} \ddot{u}_{c 1}+\sum_{n=1}^{4} k_{p n \eta 1} \delta_{p n \eta 1}+\sum_{n=1}^{4} c_{p n \eta 1} \dot{\delta}_{p n \eta 1}+k_{c u 1} u_{c 1}+c_{c u 1} \dot{u}_{c 1} \\
& \quad-k_{e u 12} \delta_{e u 12}-c_{e u 12} \dot{\delta}_{e u 12}=0
\end{aligned}
$$


The dynamic differential equation for the first-stage sun gear is

$$
\begin{aligned}
& m_{s 1} \ddot{x}_{s 1}-\sum_{n=1}^{4} k_{s p n 1} \delta_{s p n 1} \sin \psi_{s n 1}-\sum_{n=1}^{4} c_{s p n 1} \dot{\delta}_{s p n 1} \sin \psi_{s n 1} \\
& \quad+k_{s x 1} x_{s 1}+c_{s x 1} \dot{x}_{s 1}+k_{m n x} \delta_{m n x}+c_{m n x} \dot{\delta}_{m n x}=0 \\
& m_{s 1} \ddot{y}_{s 1}+\sum_{n=1}^{4} k_{s p n 1} \delta_{s p n 1} \cos \psi_{s n 1}+\sum_{n=1}^{4} c_{s p n 1} \dot{\delta}_{s p n 1} \cos \psi_{s n 1} \\
& \quad+k_{s y 1} y_{s 1}+c_{s y 1} \dot{y}_{s 1}+k_{m n y} \delta_{m n y}+c_{m n y} \dot{\delta}_{m n y}=0 \\
& \frac{I_{s 1}}{r_{s 1}^{2}} \ddot{u}_{s 1}+\sum_{n=1}^{4} k_{s p n 1} \delta_{s p n 1}+\sum_{n=1}^{4} c_{s p n 1} \dot{\delta}_{s p n 1}+k_{s u 1} u_{s 1}+c_{s u 1} \dot{u}_{s 1}+k_{m n u} \delta_{m n u}+c_{m n u} \dot{\delta}_{m n u}=0
\end{aligned}
$$

The dynamics modeling of the fixed shaft train is mainly divided into two types: reduction gears $\left(g_{7}, g_{4}, g_{3}, g_{1}\right)$ and idlers $\left(g_{7}, g_{6}, g_{5}, g_{2}\right)$. Referring to Ma et al. (2012), the dynamic equations of fixed-shaft gear train are established.

The differential equations of motion for gear $g_{8}$ are

$$
\begin{aligned}
& m_{8} \ddot{x}_{8}-k_{78} \delta_{78} \sin \psi_{78}-c_{78} \dot{\delta}_{78} \sin \psi_{78}+k_{8 x} x_{8}+c_{8 x} \dot{x}_{8}-k_{m n x} \delta_{m n x}-c_{m n x} \dot{\delta}_{m n x}=0 \\
& m_{8} \ddot{y}_{8}-k_{78} \delta_{78} \cos \psi_{78}-c_{78} \dot{\delta}_{78} \cos \psi_{78}+k_{8 y} y_{8}+c_{8 y} \dot{y}_{8}-k_{m n y} \delta_{m n y}-c_{m n y} \dot{\delta}_{m n y}=0 \\
& \frac{I_{8}}{r_{8}^{2}} \ddot{u}_{8}-k_{78} \delta_{78}-c_{78} \dot{\delta}_{78}-k_{m n u} \delta_{m n u}-c_{m n u} \dot{\delta}_{m n u}=0
\end{aligned}
$$

The differential equations of motion of the gear $g_{4}$ are

$$
\begin{aligned}
& m_{4} \ddot{x}_{4}+k_{45} \delta_{45} \sin \psi_{45}+c_{45} \dot{\delta}_{45} \sin \psi_{45}+k_{4 x} x_{4}+c_{4 x} \dot{x}_{4}+k_{34 x} \delta_{34 x}+c_{34 x} \dot{\delta}_{34 x}=0 \\
& m_{4} \ddot{y}_{4}+k_{45} \delta_{45} \cos \psi_{45}+c_{45} \dot{\delta}_{45} \cos \psi_{45}+k_{4 y} y_{4}+c_{4 y} \dot{y}_{4}+k_{34 y} \delta_{34 y}+c_{34 y} \dot{\delta}_{34 y}=0 \\
& \frac{I_{4}}{r_{4}^{2}} \ddot{u}_{4}-k_{45} \delta_{45}-c_{45} \dot{\delta}_{45}+k_{34 u} \delta_{34 u}+c_{34 u} \dot{\delta}_{34 u}=0
\end{aligned}
$$

The differential equation of motion of the gear $g_{3}$ are

$$
\begin{aligned}
& m_{3} \ddot{x}_{3}-k_{23} \delta_{23} \sin \psi_{23}-c_{23} \dot{\delta}_{23} \sin \psi_{23}+k_{3 x} x_{3}+c_{3 x} \dot{x}_{3}-k_{34 x} \delta_{34 x}-c_{34 x} \dot{\delta}_{34 x}=0 \\
& m_{3} \ddot{y}_{3}-k_{23} \delta_{23} \cos \psi_{23}-c_{23} \dot{\delta}_{23} \cos \psi_{23}+k_{3 y} y_{3}+c_{3 y} \dot{y}_{3}-k_{34 y} \delta_{34 y}-c_{34 y} \dot{\delta}_{34 y}=0 \\
& \frac{I_{3}}{r_{3}^{2}} \ddot{u}_{3}-k_{23} \delta_{23}-c_{23} \dot{\delta}_{23}-k_{34 u} \delta_{34 u}-c_{34 u} \dot{\delta}_{34 u}=0
\end{aligned}
$$

The differential equations of motion of the gear $g_{1}$ are

$$
\begin{aligned}
& m_{1} \ddot{x}_{1}+k_{12} \delta_{12} \sin \psi_{12}+c_{12} \dot{\delta}_{12} \sin \psi_{12}+k_{1 x} x_{1}+c_{1 x} \dot{x}_{1}=0 \\
& m_{1} \ddot{y}_{1}+k_{12} \delta_{12} \cos \psi_{12}+c_{12} \dot{\delta}_{12} \cos \psi_{12}+k_{1 y} y_{1}+c_{1 y} \dot{y}_{1}=0 \\
& \frac{I_{1}}{r_{1}^{2}} \ddot{u}_{1}-k_{12} \delta_{12}-c_{12} \dot{\delta}_{12}+k_{1 u} u_{1}+c_{1 u} \dot{u}_{1}=\frac{T_{1}}{r_{1}}
\end{aligned}
$$

The dynamic differential equations for the idler set are $(j=7,6,5,2)$

$$
\begin{aligned}
& m_{j} \ddot{x}_{j}+k_{j, j+1} \delta_{j, j+1} \sin \psi_{j, j+1}+c_{j, j+1} \dot{\delta}_{j, j+1} \sin \psi_{j, j+1} \\
& \quad-k_{j-1, j} \delta_{j-1, j} \sin \psi_{j-1, j}-c_{j-1, j} \dot{\delta}_{j-1, j} \sin \psi_{j-1, j}+k_{j x} x_{j}+c_{j x} \dot{x}_{j}=0 \\
& m_{j} \ddot{y}_{j}+k_{j, j+1} \delta_{j, j+1} \cos \psi_{j, j+1}+c_{j, j+1} \dot{\delta}_{j, j+1} \cos \psi_{j, j+1} \\
& \quad-k_{j-1, j} \delta_{j-1, j} \cos \psi_{j-1, j}-c_{j-1, j} \dot{\delta}_{j-1, j} \cos \psi_{j-1, j}+k_{j y} y_{j}+c_{j y} \dot{y}_{j} \\
& \frac{I_{j}}{r_{j}^{2}} \ddot{u}_{j}-k_{j, j+1} \delta_{j, j+1}-c_{j, j+1} \dot{\delta}_{j, j+1}-k_{j-1, j} \delta_{j-1, j}-c_{j-1, j} \dot{\delta}_{j-1, j}=0
\end{aligned}
$$


The differential equation of the system established by (3.1)-(3.13) is defined as

$$
\mathbf{M} \ddot{\mathbf{q}}+\mathbf{C} \dot{\mathbf{q}}+\left[\mathbf{K}_{b}+\mathbf{K}_{m}(t)\right] \mathbf{q}=\mathbf{F}(t)
$$

The coupling system is divided into the first planet stage, the second planet stage, the low speed spur gears stage and the high speed spur gears stage. The mass matrix of the system can be defined as follows. The form of $\mathbf{C}$ is determined by the Rayleigh damping (Zhang et al., 2013)

$$
\mathbf{M}=\operatorname{diag}\left(\mathbf{M}_{p 1}, \mathbf{M}_{p 2}, \mathbf{M}_{l}, \mathbf{M}_{h}\right)
$$

The generalized coordinate vectors of the system can be given as

$$
\begin{aligned}
\mathbf{q}= & \underbrace{\left[x_{c 1}, y_{c 1}, u_{c 1}, x_{r 1}, y_{r 1}, u_{r 1}, x_{s 1}, y_{s 1}, u_{s 1}, \zeta_{p 11}, \eta_{p 11}, u_{p 11}, \cdots, \zeta_{p 41}, \eta_{p 41}, u_{p 41}\right.}_{\text {First planets stage }}, \\
& \underbrace{x_{c 2}, y_{c 2}, u_{c 2}, x_{r 2}, y_{r 2}, u_{r 2}, x_{s 2}, y_{s 2}, u_{s 2}, \zeta_{p 12}, \eta_{p 12}, u_{p 12}, \cdots, \zeta_{p 42}, \eta_{p 42}, u_{p 42}}_{\text {Second planets stage }}, \\
& \underbrace{x_{8}, y_{8}, u_{8}, x_{7}, y_{7}, u_{7}, x_{6}, y_{6}, u_{6}, x_{5}, y_{5}, u_{5}, x_{4}, y_{4}, u_{4}}_{\text {High speed spur gears stage }},
\end{aligned}
$$

The external load vector of the system is given as follows

$$
\mathbf{F}(t)=[\underbrace{0,0, \ldots, 0}_{21}, 0,0,-\frac{T_{c 2}}{r_{c 2}}, 0,0, \ldots, 0,0, \frac{T_{1}}{r_{1}}]^{\mathrm{T}}
$$

The support stiffness matrix of the system is defined as follows

$$
\mathbf{K}_{b}=\operatorname{diag}\left(\mathbf{K}_{b p 1}, \mathbf{K}_{b p 2}, \mathbf{K}_{b l}, \mathbf{K}_{b h}\right)
$$

The time-varying meshing stiffness matrix and the inter-stage coupling stiffness matrix of the system can be written as

$$
\mathbf{K}_{m}(t)=\left[\begin{array}{cccc}
\mathbf{K}_{m p 1}(t) & \mathbf{K}_{p 1 p 2} & \mathbf{K}_{p 1 l} & \mathbf{0} \\
& \mathbf{K}_{m p 2}(t) & \mathbf{0} & \mathbf{0} \\
& & \mathbf{K}_{m l}(t) & \mathbf{K}_{l h} \\
\text { sym } & & & \mathbf{K}_{m h}(t)
\end{array}\right]
$$

\section{Study on dynamical behavior}

The gear parameters of the multistage gear transmission system are derived from the cutting unit of a continuous miner in the National Coal Mine Energy Mining Equipment Test Center. The shearer has a cutting speed of $29 \mathrm{rpm}$, cutting depth of $300 \mathrm{~mm}$ and traction speed of $1.5 \mathrm{~m} / \mathrm{min}$ during the test. Table 1 is the parameter of the high-speed and low-speed gear subsystem, and Table 2 is the parameter of the two-stage planetary gears subsystem. $f_{m 1}(180 \mathrm{~Hz}), f_{m 2}(30 \mathrm{~Hz})$, $f_{n 1}(687 \mathrm{~Hz}), f_{n 2}(464 \mathrm{~Hz})$ represent the meshing fundamental frequencies of the first planet stage, second planet stage, high speed spur gears stage and low speed spur gears stage, respectively. 
Table 1. Parameters of high-speed and low-speed spur gears subsystems

\begin{tabular}{|l|c|c|c|c|c|c|c|c|}
\hline & Gear 1 & Gear 2 & Gear 3 & Gear 4 & Gear 5 & Gear 6 & Gear 7 & Gear 8 \\
\hline \hline Tooth number & 28 & 39 & 40 & 27 & 33 & 33 & 33 & 40 \\
\hline Module [mm] & 8 & 8 & 8 & 10 & 10 & 10 & 10 & 10 \\
\hline Mass [kg] & 13.5 & 27.1 & 28.4 & 25.1 & 35.1 & 35.1 & 35.1 & 52.4 \\
\hline Inertia $\left[\mathrm{kg} \mathrm{m}{ }^{2}\right]$ & 0.085 & 0.16 & 0.38 & 0.22 & 0.52 & 0.52 & 0.52 & 1.04 \\
\hline Bearing stiffness $[\mathrm{N} / \mathrm{m}]$ & $5 \cdot 10^{8}$ & $5 \cdot 10^{8}$ & $5 \cdot 10^{8}$ & $10^{9}$ & $10^{9}$ & $10^{9}$ & $10^{9}$ & $10^{9}$ \\
\hline
\end{tabular}

Table 2. Gear parameters of the two-stage planetary subsystem

\begin{tabular}{|l|c|c|c|c|c|}
\hline & Stage & Sun & Planet & Ring & Carrier \\
\hline \hline \multirow{2}{*}{ Tooth number } & First & 18 & 31 & 82 & - \\
\cline { 2 - 6 } & Second & 18 & 23 & 66 & - \\
\hline \multirow{2}{*}{$\begin{array}{l}\text { Module } \\
{[\mathrm{mm}]}\end{array}$} & First & 7 & 7 & 7 & - \\
\cline { 2 - 6 } $\begin{array}{l}\text { Mass } \\
{[\mathrm{kg}]}\end{array}$ & Second & 11 & 11 & 11 & - \\
\hline $\begin{array}{l}\text { Inertia } \\
{\left[\mathrm{kg} \mathrm{m}{ }^{2}\right]}\end{array}$ & First & 4.5 & 12.3 & 32.9 & 95.6 \\
\cline { 2 - 6 } & Second & 10.1 & 28.7 & 108.6 & 706.3 \\
\hline $\begin{array}{l}\text { Torsional } \\
\text { stiffness }[\mathrm{Nm} / \mathrm{rad}]\end{array}$ & First & 0.035 & 0.072 & 1.36 & 1.03 \\
\cline { 2 - 6 } & Second & 0.06 & 0.23 & 7.11 & 22.9 \\
\cline { 2 - 6 } $\begin{array}{l}\text { Support } \\
\text { stiffness }[\mathrm{N} / \mathrm{m}]\end{array}$ & Second & $4 \cdot 10^{9}$ & - & $4 \cdot 10^{9}$ & $4 \cdot 10^{9}$ \\
\cline { 2 - 6 } & First & $5 \cdot 10^{8}$ & $5 \cdot 10^{8}$ & $5 \cdot 10^{8}$ & $5 \cdot 10^{8}$ \\
\hline
\end{tabular}

\subsection{Model verification}

The variable mesh stiffness of the coupled system is solved by referring to Parker (2002), and the phase relationship of the planetary system is solved by referring to Parker and Lin (2004). The dynamical differential equation of the multistage gear transmission system is simulated by the Newmark- $\beta$ method. The calculated values are compared and verified with test data collected by the continuous miner test system in "National Coal Mine Energy Mining Equipment Test Center". Figure 5 is the test site of acceleration for the multistage gear systems of the shearer. The measuring point is located on the bearing housing of the coupling shaft where gear $g_{8}$ is installed, that is, the connecting shaft of the planetary subsystem and the fixed shaft gears subsystem.

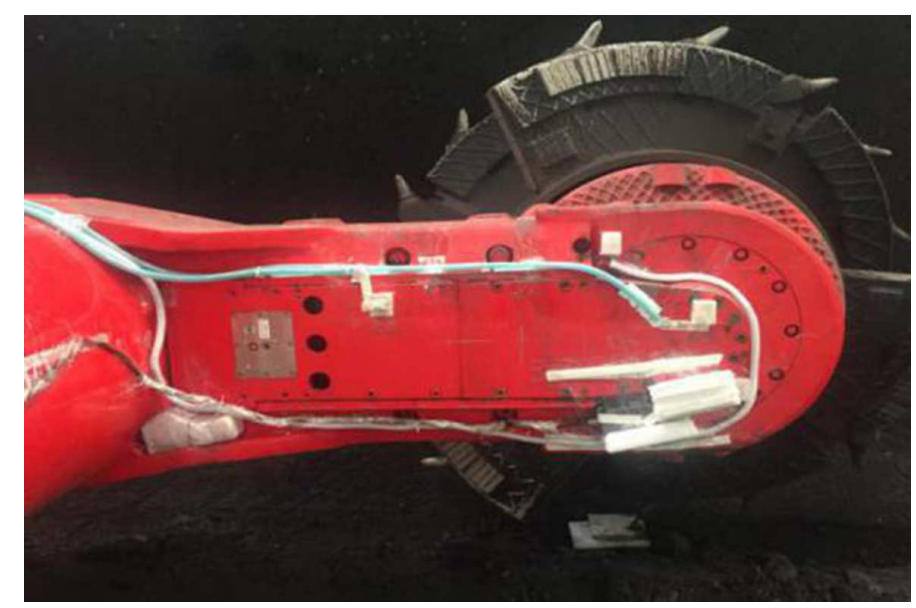

Fig. 5. Acceleration test site of the multistage gear transmission system 
Figure 6 shows a time domain comparison between the calculated and measured values of the vibration acceleration of gear $g_{8}$ in the $x$ direction. The amplitudes of both are within the range of $\pm 0.6 \mathrm{~m} / \mathrm{s}^{2}$. The simulation results are in good agreement with the experimental values. Figure 7 shows a frequency domain comparison between the calculated and measured values of the acceleration of gear $g_{8}$ in the $x$ direction. Gear $g_{8}$ is located in the low speed spur gears stage, and its vibration acceleration is mainly generated by the meshing frequency $f_{n 2}$ and its harmonic component of the low speed spur gears stage. The frequency domain results of Fig. 7 show that $f_{n 2}, 2 f_{n 2}, 3 f_{n 2}, \ldots, 7 f_{n 2}$ are the most important frequency components of $g_{8}$ acceleration. The high-speed meshing frequencies $f_{n 1}, 2 f_{n 1}, 3 f_{n 1}$ also have a certain excitation effect on $g_{8}$ acceleration. Figure 7 shows that the first stage planetary meshing frequency $f_{m 1}$ and its multiplication have a stronger influence on the acceleration calculated value than the experimental value. This is due to the installation position of acceleration test points. The accelerometer of gear $g_{8}$ is installed on the side of spur gear transmission, which weakens the reception effect of the planetary acceleration signal. The acceleration test values in Fig. 7 have different degrees of side bands near the meshing frequency, which is caused by the joint excitation of the meshing frequency and the rotating shaft frequency. The theoretical model does not take into account the influence of the rotating shaft frequency, so it does not produce side bands.

(a)

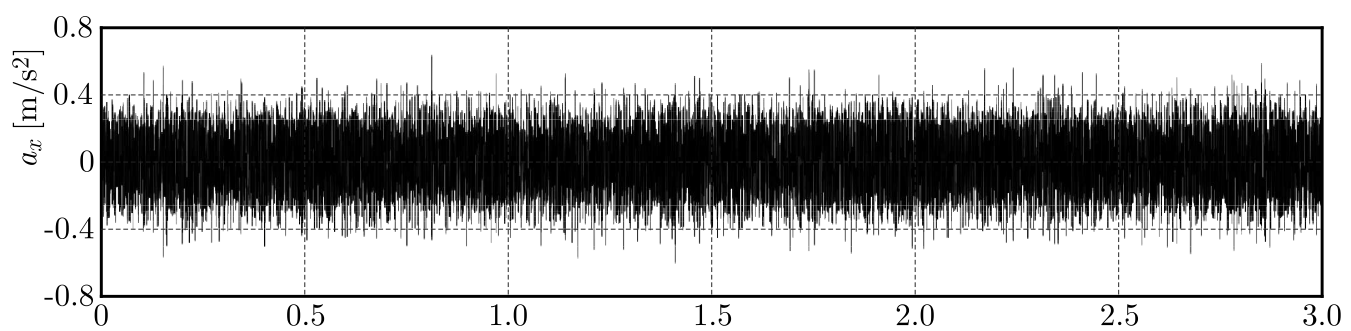

(b)

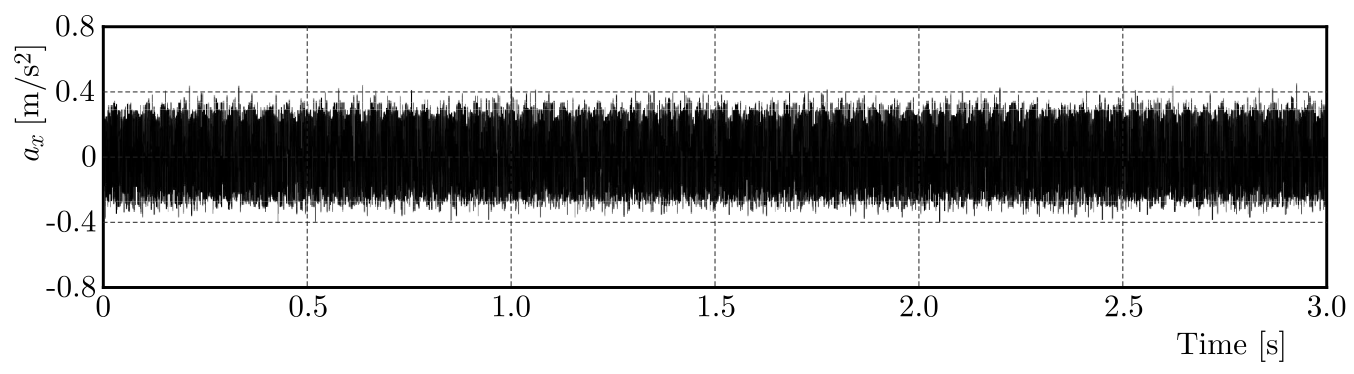

Fig. 6. Time domain comparison between calculated and measured values of acceleration of $g_{8}$

\subsection{Effect of coupling shaft radius on acceleration of the planet system}

The carrier in the first stage planet subsystem and the sun in second stage planet subsystem are connected by a coupling shaft. The theoretical model fully considers the influence of the change of coupling shaft radius $r_{e}$ and first stage carrier radius $r_{c 1}$ on the dynamic behavior of two stage planet systems.

Figure 8 shows frequency spectra of the acceleration of the sun and the planet located in the second planet stage in the $u$ direction when $r_{e}\left(r_{e}=0.5 r_{s 2}-3.0 r_{s 2}\right)$ changes $\left(r_{c 1}=3 r_{s 2}\right.$ remains unchanged at this time). When $r_{e}$ changes from $0.5 r_{s 2}$ to $3.0 r_{s 2}$ ( $r_{s 2}$ is constant value), with the increase of $r_{e}$, the amplitude of the second stage planetary meshing frequency $f_{m 2}$ and its harmonic frequencies $3 f_{m 2}, 5 f_{m 2}, 7 f_{m 2}$ and the coupling frequency $2 f_{m 1} \pm f_{m 2}, 6 f_{m 1} \pm f_{m 2}$ are continuously decreasing, while the amplitude of the first stage planetary meshing frequency $f_{m 1}$ and its harmonic frequency $3 f_{m 1}, 5 f_{m 1}, 7 f_{m 1}$ are constantly rising. It shows that with the increase of size $r_{e}$, the influence of $f_{m 1}$ on the acceleration of the secondary planet is enhanced, and the 
(a)

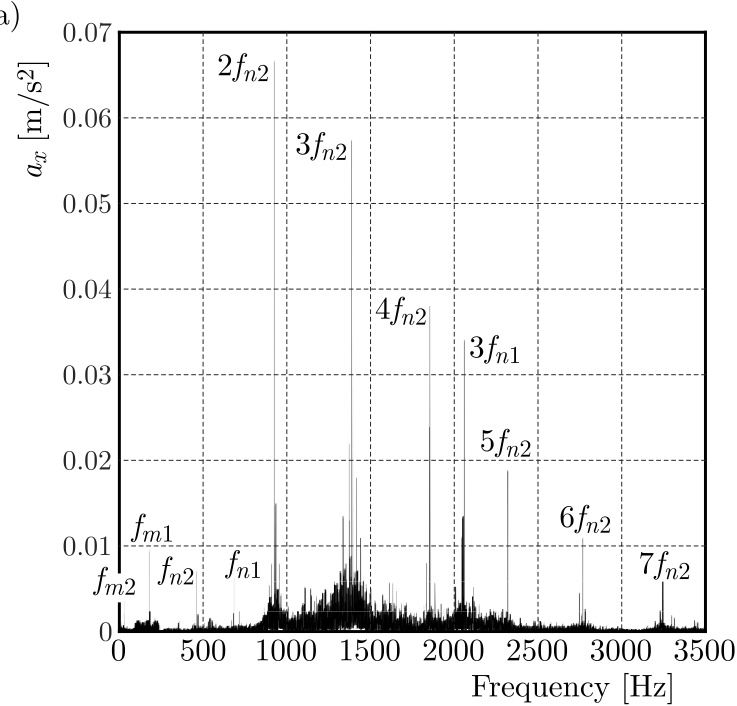

(b)

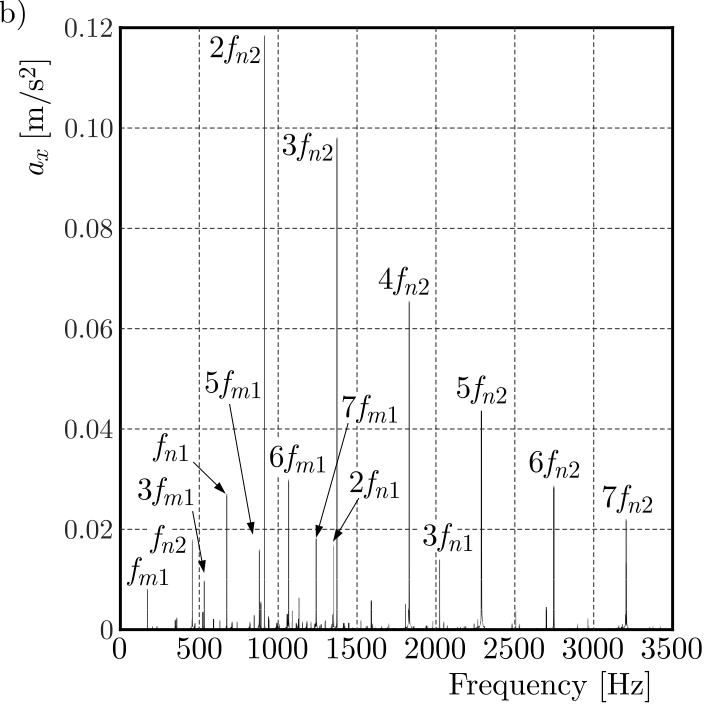

Fig. 7. Frequency domain comparison between calculated and measured values of acceleration of $g_{8}$

(a)

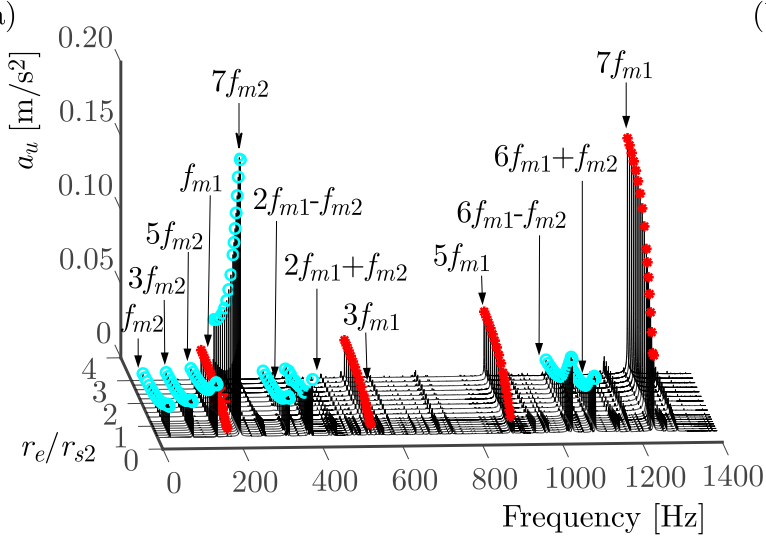

(b)

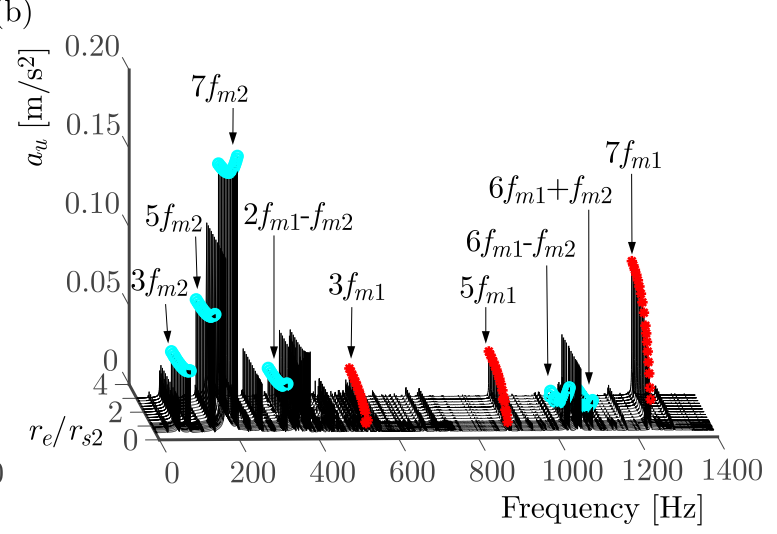

Fig. 8. Frequency spectra of acceleration of the second stage planets with different coupling radius

excitation of $f_{m 2}$ on the acceleration of the secondary planet is suppressed. That is, as the size of $r_{e}$ increases, the coupling effect between the planetary systems is enhanced.

Figure 9 displays a relationship between acceleration and time of the sun gear located in the second planet stage in the $u$ direction when $r_{e}\left(r_{e}=0.5 r_{s 2}, 1.1 r_{s 2}, 3 r_{s 2}\right)$ changes. With the increase of $r_{e}$, the amplitude of $a_{u}$ does not change significantly, but the period of $a_{u}$ changes from $t_{c 2} / 4$ to $t_{c 1} / 4$ ( $t_{c 2}$ is the rotation period of the second stage carrier, and $t_{c 1}$ is the rotation period of the first stage carrier). This phenomenon can be explained by the frequency domain distribution of $a_{u}$ in Fig. 8, with the increase of $r_{e}$, the main components of the mesh frequency gradually change from $f_{m 2}$ to $f_{m 1}$, while the change of $r_{e}$ could not cause obvious change of the vibration level for planet systems.

Figure 10 shows frequency spectra of the acceleration of the second stage sun gear and the planet in the $u$ direction when $r_{c 1}\left(r_{c 1}=r_{s 2}-3.0 r_{s 2}\right)$ changes $\left(r_{e}=r_{s 2}\right.$ remains unchanged at this time). When $r_{c 1}$ changes from $r_{s 2}$ to $3.0 r_{s 2}$ ( $r_{s 2}$ is constant value), with the increase of $r_{c 1}$, the amplitude of the second stage planetary meshing frequency $f_{m 2}$ and its harmonic frequencies $3 f_{m 2}, 5 f_{m 2}$ and the coupling frequency $2 f_{m 1} \pm f_{m 2}, 6 f_{m 1} \pm f_{m 2}$ does not change significantly or has decreased slightly, while the amplitude of the first stage planetary meshing frequency $f_{m 1}$ 
(a) $r_{e}=0.5 r_{s 2}$

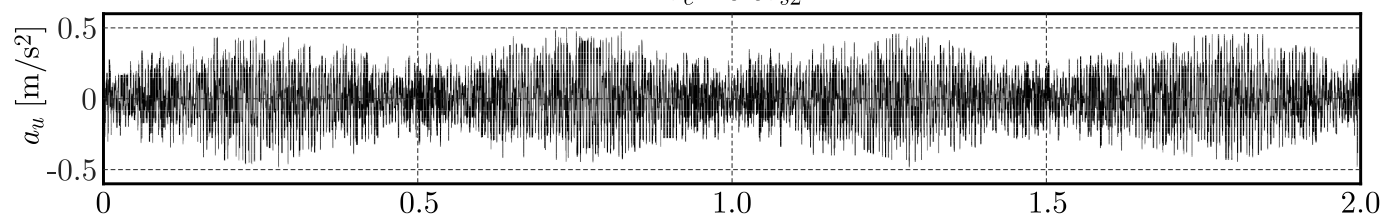

(b)

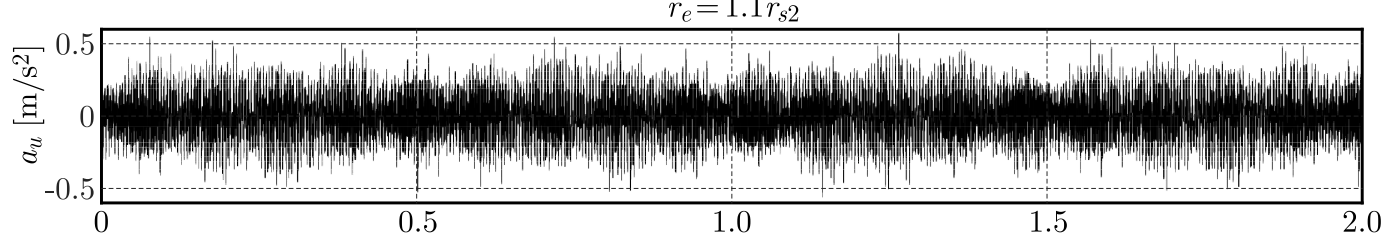

(c)

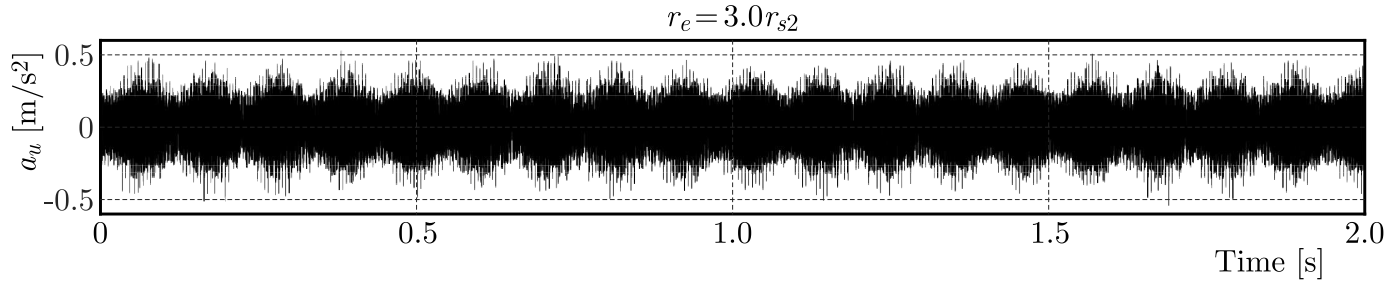

Fig. 9. The relationship between $a_{u}$ and time of the second stage sun with different coupling radius

(a)

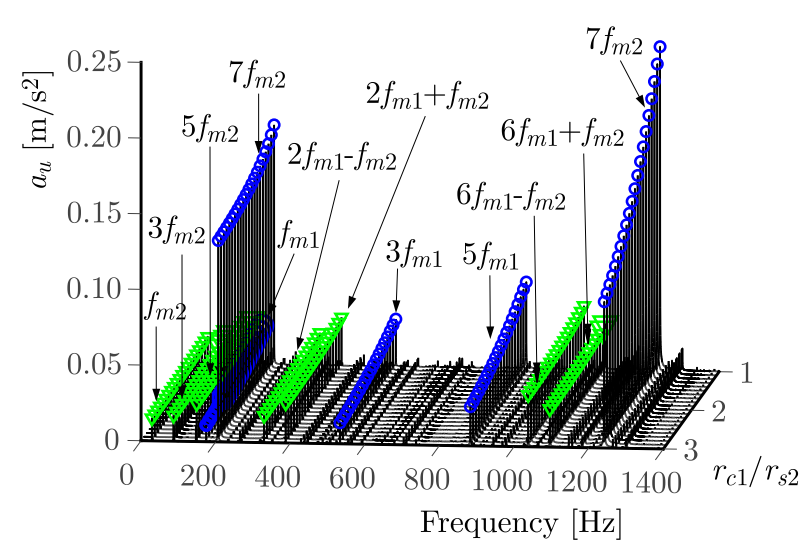

(b)

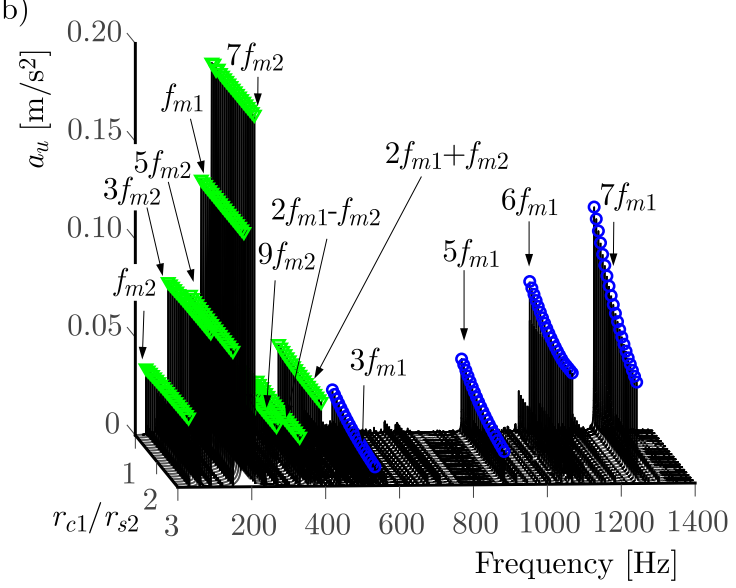

Fig. 10. Frequency spectra of the acceleration of the second stage sun gear and the planet in the $u$ direction

and its harmonic frequency $3 f_{m 1}, 5 f_{m 1}, 7 f_{m 1}$ are gradually decreasing. For the second stage planetary system response, it shows that the change of $r_{c 1}$ mainly affects the first stage meshing frequency $f_{m 1}$, and the influence on the second stage meshing frequency $f_{m 2}$ is relatively small.

Figure 11 presents the relationship between acceleration and time of the second stage sun gear in the $u$ direction when $r_{c 1}\left(r_{c 1}=r_{s 2}, 1.5 r_{s 2}, 3 r_{s 2}\right)$ changes. With the increase of $r_{c 1}$, the amplitude of acceleration decreases gradually. It shows that the change of $r_{c 1}$ causes a change of the vibration level for the planet systems. When $r_{c 1}=r_{s 2}$, that is, when the size of $r_{c 1}$ is not taken into account in the coupling model, the vibration level of the system is the highest. Therefore, most of the multistage gear vibration models without considering the influence of the radius of the coupling shaft are conservative calculation methods. Considering the influence of the actual size of $r_{c 1}$ is beneficial to the lightweight design of multistage gear systems. 


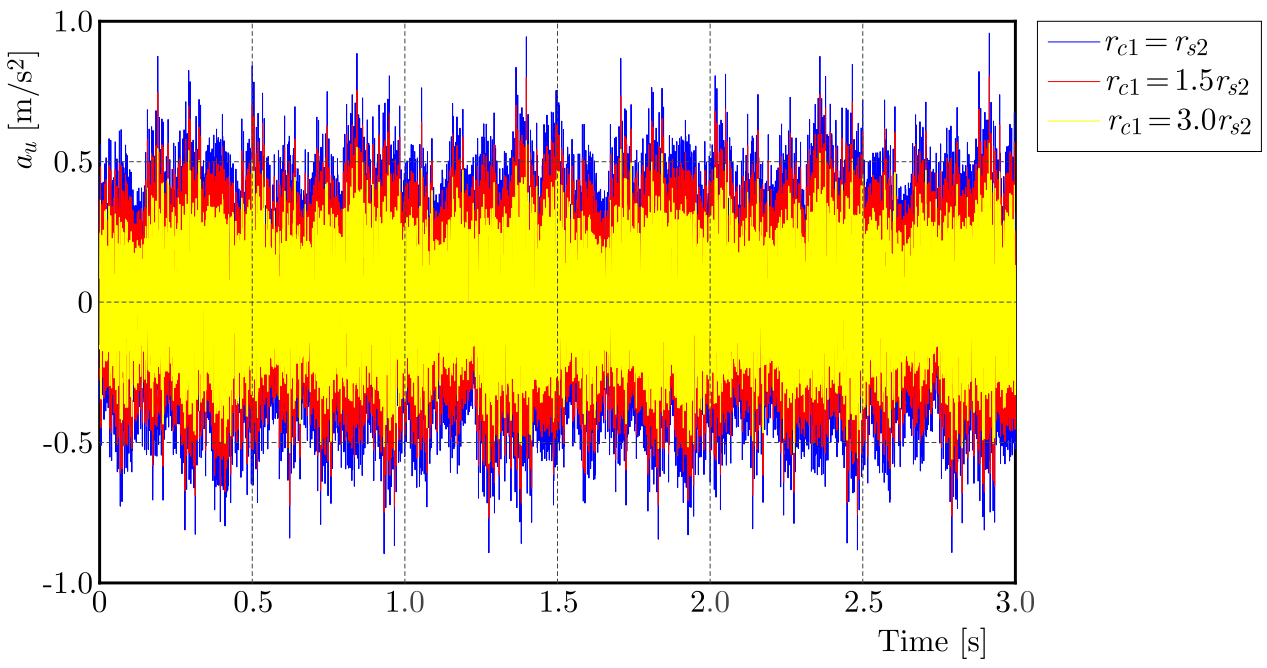

Fig. 11. The relationship between acceleration and time of the second stage sun gear in the $u$ direction

\section{Conclusion}

Considering the influence of the cross-section size of the coupling shaft, the coupling shaft is regarded as a translational torsion spring, and the coupling analytical model of two-stage planets and a multistage parallel shaft gear is developed by the lumped parameter method. The coupling model fully considers the effect of the cross-section radius of the coupling shaft on the multistage gear system. The acceleration test of gears further verifies the accuracy of the theoretical model and lays a foundation for the study of the dynamic behavior of the system.

The research on the influence of coupling shaft radius on the acceleration of the planetary system shows that with the increase of cross-section size of the coupling shaft, the influence of the first stage meshing frequency on the second stage planetary system is enhanced, while the excitation effect of the second stage meshing frequency is suppressed. That is to say, with the increase of cross-section size of the coupling shaft, the coupling effect between planetary systems is enhanced. The acceleration level of the planetary system decreases gradually with the increase of radius of the first stage planet carrier. The influence of the actual size of the planet carrier when coupling can be considered beneficial in reducing the vibration level in a multistage gear system.

\section{Acknowledgement}

The paper is completed with the support of Chinese National Natural Science Foundation (U1708254) and National Natural Science United Foundation of China (U1710119).

\section{References}

1. BAI W., Qin D., WANG Y., Lim T.C., 2018, Dynamic characteristics of motor-gear system under load saltations and voltage transients, Mechanical Systems and Signal Processing, 100, 1-16

2. Chowdhury S., Yedavalli R.K., 2017, Dynamics of low speed geared shaft systems mounted on rigid bearings, Mechanism and Machine Theory, 112, 123-144

3. Chowdhury S., Yedavalli R.K., 2018, Vibration of high speed helical geared shaft systems mounted on rigid bearings, International Journal of Mechanical Sciences, 142-143, 176-190

4. Eritenel T., Parker R.G., 2009, Modal properties of three-dimensional helical planetary gears, Journal of Sound and Vibration, 325, 1, 397-420 
5. Guo Y., PArker R.G., 2010, Dynamic modeling and analysis of a spur planetary gear involving tooth wedging and bearing clearance nonlinearity, European Journal of Mechanics, 29, 6, 1022-1033

6. Guo Y., PArker R.G., 2012, Dynamic analysis of planetary gears with bearing clearance, Journal of Computational and Nonlinear Dynamics, 7, 4, 1-13

7. Hammami A., Fernandez del Rincon A., Chaari F., Iglesias Santamaria M., Viadero Rueda F., HAdDAR M., 2016, Effects of variable loading conditions on the dynamic behaviour of planetary gear with power recirculation, Measurement, 94, 306-315

8. Hammami A., Fernandez del Rincon A., ChaAri F., Viadero Rueda F., Haddar M., 2015a, Dynamic behaviour of back to back planetary gear in run up and run down transient regimes, Journal of Mechanics, 31, 4, 481-491

9. Hammami A., Rincon A.F., del Rueda F.V., Chaari F., Haddar M., 2015b, Modal analysis of back-to-back planetary gear: experiments and correlation against lumped parameter model, Journal of Theoretical and Applied Mechanics, 53, 1, 125-138

10. Karray M., Chaari F., del Rincon A.F., Viadero F., Haddar M., 2013, An experimental investigation of the dynamic behavior of planetary gear set, [In:] Design and Modeling of Mechanical Systems, LNME, M. Haddar et al. (Edit.), 199-206

11. Kubur M., Kahraman A., Zini D.M., Kienzle K., 2004, Dynamic analysis of a multi-shaft helical gear transmission by finite elements: model and experiment, Journal of Vibration and Acoustics, 126, 3, 398-406

12. Lin J., PARker R.G., 1999, Analytical characterization of the unique properties of planetary gear free vibration, Journal of Vibration and Acoustics, 121, 3, 316-321

13. Lin J., PARker R.G., 2002, Mesh stiffness variation instabilities in two-stage gear systems, Journal of Vibration and Acoustics, 124, 68-76, DOI: 10.1115/1.1424889

14. LiU C., Qin D., LiaO Y., 2016, Dynamic analysis for the cutting electromechanical transmission system in the long-wall Shearer, Journal of Mechanical Engineering, 52, 7, 14-22

15. LiU C., Qin D., LiaO Y., 2017. Dynamic modeling and analysis of high-speed planetary gear including centrifugal force, Journal of the Brazilian Society of Mechanical Sciences and Engineering, 39, 10, 3769-3778

16. Liu C., Qin D., Lim T.C., LiaO Y., 2014, Dynamic characteristics of the herringbone planetary gear set during the variable speed process, Journal of Sound and Vibration, 333, 24, 6498-6515

17. Ma H., WAng Q.B., HuAng J., 2013, Vibration response analysis of gear coupled rotor system considering geometric eccentric effect of helical gears, Journal of Aerospace Power, 28, 1, 16-24

18. MA H., ZHU L.S., WANG Q.B., 2012, Modal coupling characteristic analysis of a helical gear rotor system with parallel shafts, Proceedings of the CSEE, 32, 29, 131-136

19. PArker R.G., Lin J., 2004, Mesh phasing relationships in planetary and epicyclic gears, Journal of Mechanical Design, 126, 365-370

20. Wei J., Zhang A., Qin D., Lim T.C., Shu R., Lin X., Meng F., 2017, A coupling dynamics analysis method for a multistage planetary gear system, Mechanism and Machine Theory, 110, 27-49

21. Yi Y.Y., QIN D., LiU Z., 2018, Investigation of electromechanical coupling vibration characteristics of an electric drive multistage gear system, Mechanism and Machine Theory, 121, 446-459

22. Zhang Y., Wang Q., Ma H., HuAng J., ZhaO C., 2013, Dynamic analysis of three-dimensional helical geared rotor system with geometric eccentricity, Journal of Mechanical Science and Technology, 27, 11, 3231-3242

23. Zhang L., Wang Y., Wu K., Sheng R., 2017, Three-dimensional modeling and structured vibration modes of two-stage helical planetary gears used in cranes, Shock and Vibration, 2017, $1-18$ 